International Journal of Pure and Applied Mathematics

Volume 115 No. 4 2017, 719-729

ISSN: 1311-8080 (printed version); ISSN: 1314-3395 (on-line version)

url: http://www.ijpam.eu

doi: 10.12732 /ijpam.v115i4.6

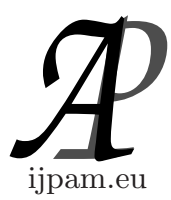

\title{
STURM-LIOUVILLE DIFFERENTIAL EQUATION WITH NON-LOCAL BOUNDARY CONDITIONS
}

\author{
A.M.A. El-Sayed ${ }^{1}$, F.A. EL-Raheem ${ }^{2}$, A.O. Buhalima ${ }^{3} \S$ \\ ${ }^{1}$ Faculty of Science \\ Alexandria University \\ Alexandria, EGYPT \\ ${ }^{2}$ Faculty of Education \\ Alexandria University \\ Alexandria, EGYPT \\ ${ }^{3}$ Faculty of Science \\ Omar AlMukhtar University \\ Albeda, LIBYA
}

\begin{abstract}
We investigate the existence and some general properties of eigenvalues and eigenfunctions of a nonlocal boundary value problem of the Sturm-Liouville differential equation.
\end{abstract}

AMS Subject Classification: 34A55, 34B10, 34B15, 34B18, 34L10

Key Words: Sturm-Liouville boundary value problem, nonlocal condition, eigenvalues, eigenfunctions

\section{Introduction}

Many interesting applications of differential equations arise in recent years with nonlocal condition often appear in Mathematics, mechanics, physics, geophysics and other branches of natural sciences (see [1]-[5] and [10]-[13]). Afterwards

Received: December 29, 2016

Revised: $\quad$ March 21, 2017

Published: $\quad$ August 7, 2017

(c) 2017 Academic Publications, Ltd. url: www.acadpubl.eu

${ }^{\S}$ Correspondence author 
the number of differential problems with nonlocal boundary conditions had increased. Quite new area, related to problems of this type, deals with investigation of the spectrum of Sturm-Liouville with nonlocal conditions.

Recently, in ([6]-[8]) the authors studies the existence and some asympotic properties of the eigenvalues and eigenfunctions of the boundary value problem of the Sturm- Liouville differential equation

$$
-y^{\prime \prime}+q(x) y=\lambda^{2} y, \quad 0 \leq x \leq \pi,
$$

with different kinds of nonlocal boundary conditions.

Consider the nonlocal boundary value problem of the Sturm-Liouville equation (1) with the non-local conditions

$$
y^{\prime}(0)-H y(0)=0, y(\xi)=0, \quad \xi \in(0, \pi],
$$

where the non-negative real function $q(x)$ has a second piecewise integrable derivatives on $(0, \pi), H$ is real and $\lambda$ is spectral parameter.

Here we study the existence and some general properties of the eigenvalues and eigenfunctions of the two non-local boundary value problems (1) and (2). Comparison with the local boundary value problem problem of equation (1) with the local boundary value problem

$$
y^{\prime}(0)-H y(0)=0, \quad y(\pi)=0
$$

will be given.

\section{General Properties}

Here we prove some results concerning the eigenvalues and eigenfunctions of the nonlocal problem (1)-(2).

Lemma 1. The eigenvalues of the nonlocal boundary value problem (1) and (2) are real.

Proof. Let $y_{0}(x)$ be the eigenfunction that corresponds to the eigenvalue $\lambda_{0}$ of the problem (1) and (2), then

$$
-y_{0}^{\prime \prime}+q(x) y_{0}=\lambda_{0}^{2} y_{0} \quad(0 \leq x \leq \pi),
$$

and

$$
y_{0}(0)-H y_{0}(0)=y_{0}(\xi)=0 \text {. }
$$


Multiplying both sides of (3) by $\overline{y_{0}}$ and then integrating form 0 to $\xi$ with respect to $x$, we have

$$
-\left.\bar{y}_{0} y_{0}\right|_{0} ^{\xi}+\int_{0}^{\xi}\left|y_{0}\right|^{2} d x+\int_{0}^{\xi} q(x)\left|y_{0}\right|^{2} d x=\lambda_{0}^{2} \int_{0}^{\xi}\left|y_{0}\right|^{2} d x .
$$

Using the boundary condition (4), we have

$$
\lambda_{0}^{2}=\frac{\int_{0}^{\xi}\left[q(x)\left|y_{0}\right|^{2}+\left|y_{0}^{\prime}\right|^{2}\right] d x+H}{\int_{0}^{\xi}\left|y_{0}\right|^{2} d x} .
$$

From which it follows the reality of $\lambda_{0}^{2}$.

Lemma 2. The eigenfunctions that corresponds to two different eigenvalues of the non-local boundary value problem (1) and (2) are orthogonal.

Proof. Let $\lambda_{1} \neq \lambda_{2}$ be two different eigenvalues of the non-local boundary value problem (1) and (2). Let $y_{1}(x), y_{2}(x)$ be the corresponding eigenfunctions, then

$$
\begin{gathered}
-y_{1}^{\prime \prime}+q(x) y_{1}=\lambda_{1}^{2} y_{1} \quad(0 \leq x \leq \pi) \\
y_{1}(0)-H y_{1}(0)=y_{1}(\xi)=0
\end{gathered}
$$

and

$$
\begin{gathered}
-y_{2}^{\prime \prime}+q(x) y_{2}=\lambda_{2}^{2} y_{2} \quad(0 \leq x \leq \pi), \\
y_{2}(0)-H y_{2}(0)=y_{2}(\xi)=0
\end{gathered}
$$

Multiplying both sides of (5) by $\bar{y}_{2}$ and integrating with respect to $x$, we obtain

$$
-\int_{0}^{\xi} y^{\prime \prime}{ }_{1} \bar{y}_{2} d x+\int_{0}^{\xi} q(x) y_{1} \bar{y}_{2} d x=\lambda_{1}^{2} \int_{0}^{\xi} y_{1} \bar{y}_{2} d x
$$

by taking the complex conjugate of (7) and multiply it by $y_{1}$ and integrate the resulting expression with respect to $x$, we have

$$
-\int_{0}^{\xi} y_{1} \bar{y}^{\prime \prime}{ }_{2} d x+\int_{0}^{\xi} q(x) y_{1} \bar{y}_{2} d x=\lambda_{1}^{2} \int_{0}^{\xi} y_{1} \bar{y}_{2} d x .
$$

Subtracting (9) from (10) and using the boundary conditions of (6) and (8) we obtain

$$
\left(\lambda_{1}^{2}-\lambda_{2}^{2}\right) \int_{0}^{\xi} y_{1} \bar{y}_{2} d x=0, \quad \lambda_{1}^{2} \neq \lambda_{2}^{2}
$$

which completes the proof. 


\section{The Asymptotic Formulas for the Solution}

Here we study the asymptotic formulas for the solutions of problem (1) and (2).

Lemma 1.1 deals with the nature the eigenvalues. Let be $\phi(x, \lambda)$ the solution of equation (1) and (2) satisfying the initial conditions

$$
\phi(0, \lambda)=1, \quad \phi^{\prime}(0, \lambda)=H
$$

and by $\vartheta(x, \lambda)$ the solution of the same equation, satisfying the initial conditions

$$
\vartheta(0, \lambda)=0, \quad \vartheta^{\prime}(0, \lambda)=1 .
$$

We notes that $\phi(x, \lambda)$ and $\vartheta(x, \lambda)$ are linearly independent if and only if $\omega(\lambda) \neq$ 0 .

$$
\omega(\lambda)=\phi(x, \lambda) \vartheta^{\prime}(x, \lambda)-\phi^{\prime}(x, \lambda) \vartheta(x, \lambda) .
$$

The solution

$$
Y(x, \lambda)=\alpha \phi(x, \lambda)+\beta \vartheta(x, \lambda), \text { atleast } \alpha \text { or } \beta \neq 0 .
$$

$Y(x, \lambda)$ as eigenfunction must satisfy the first condition (2), we have

$$
y^{\prime}(0, \lambda)-H y(0, \lambda)=0,
$$

and then,

$$
\alpha \phi^{\prime}(0, \lambda)+\beta \vartheta^{\prime}(0, \lambda)-H(\alpha \phi(0, \lambda)+\beta \vartheta(0, \lambda))=0 .
$$

After using the condition (11), (12), we get

$$
\alpha \phi(\xi, \lambda)=0, \text { where } \alpha \neq 0,
$$

therefore, The characteristic equation will be

$$
\omega(\lambda)=\phi(\xi, \lambda) .
$$

Lemma 3. The solution $\phi(x, \lambda)$ of problem (1) and (2) satisfy the integral equations

$$
\phi(x, \lambda)=\cos \lambda x+\frac{H}{\lambda} \sin \lambda x+\int_{0}^{x} \frac{\sin \lambda(x-\tau)}{\lambda} q(\tau) \phi(\tau, \lambda) d \tau .
$$


Proof. First we obtain formula (14) Indeed, with solution of the form $q(x)=$ 0 . (1) becomes becomes $-y^{\prime \prime}=\lambda^{2} y$ by means of variation of parameter method, we have

$$
\phi(x, \lambda)=C_{1}(x, \lambda) \cos \lambda x+C_{2}(x, \lambda) \sin \lambda x
$$

and the direct calculation of $C_{1}(x, s)$ and $C_{2}(x, s)$, we have

$$
\begin{aligned}
& C_{1}(x, \lambda)=1-\int_{0}^{x} \frac{\sin \lambda \tau}{\lambda} q(\tau) \phi(\tau, \lambda) d \tau, \\
& C_{2}(x, \lambda)=\frac{H}{\lambda}+\int_{0}^{x} \frac{\cos \lambda \tau}{\lambda} q(\tau) \phi(\tau, \lambda) d \tau,
\end{aligned}
$$

substituting from (16) into (15) equation (14) follows. Second we show that the integral representation (14) satisfies the problem (1) and (11). Let $\varphi(x, \lambda)$ be the solution of (1), so that

$$
q(x) \phi(x, \lambda)=\phi^{\prime \prime}(x, \lambda)+\lambda^{2} \phi(x, \lambda) .
$$

We multiply both sides by

$$
\frac{\sin \lambda(x-\tau)}{\lambda}
$$

and integrating with respect to $\tau$ from 0 to $x$ we obtain

$$
\begin{aligned}
\int_{0}^{x} \frac{\sin \lambda(x-\tau)}{\lambda} q(\tau) \phi(\tau, \lambda) d \tau=\int_{0}^{x} \frac{\sin \lambda(x-\tau)}{\lambda} \phi^{\prime \prime}(\tau, \lambda) d \tau \\
+\lambda^{2} \int_{0}^{x} \frac{\sin \lambda(x-\tau)}{\lambda} \phi(\tau, \lambda) d \tau
\end{aligned}
$$

Integrating by parts twice and using the condition (11), we have

$$
\begin{aligned}
& \int_{0}^{x} \frac{\sin \lambda(x-\tau)}{\lambda} \phi^{\prime \prime}(\tau, \lambda) d \tau \\
& \quad=\phi(x, \lambda)-\frac{H}{\lambda} \sin \lambda x-\cos \lambda x-\lambda \int_{0}^{x} \sin \lambda(x-\tau) \phi(\tau, \lambda) d \tau .
\end{aligned}
$$

By substituting from (18) into (17) we get the required formula (14).

Lemma 4. Let $\lambda=\sigma+i t$, then there exists $\lambda_{0}>0$, such that $|\lambda|>\lambda_{0}$ the following relation of the non-local boundary value problem (1) and (2) holds true

$$
\phi(x, \lambda)=\cos \lambda x+O\left(\frac{e^{|t| x}}{|\lambda|}\right) .
$$


Proof. We show first that

$$
\phi(x, \lambda)=O\left(e^{|t| x}\right)
$$

where the inequality is uniformly with respect to $x$. Form the integral equation (14) we have

$$
|\phi(x, \lambda)| \leq e^{|t|(x)}+\frac{|H|}{|\lambda|} e^{|t| x}+\frac{1}{\lambda} \int_{0}^{x} e^{|t| x}|q(\tau)||\phi(\tau, \lambda)| d \tau .
$$

By using the notation $\phi(x, \lambda) e^{-|t| x}=F(x, \lambda)$, equation (20) takes the form

$$
|F(x, \lambda)| \leq 1+\frac{|H|}{|\lambda|}+\int_{0}^{\pi} \frac{|q(\tau)|}{|\lambda|}|F(\tau, \lambda)| d \tau .
$$

Let $\mu=\max _{0 \leq x \leq \pi} F(x, \lambda)$, so that from (21) it follows that

$$
\mu \leq \frac{1+\frac{|H|}{|\lambda|}}{1-\frac{1}{\lambda} \int_{0}^{\pi}|q(\tau)| d \tau}
$$

For $|\lambda|>\lambda_{0}=\int_{0}^{\pi}|q(\tau)| d \tau$ it follows from the last inequality that $F(x, \lambda) \leq$ constant $/|\lambda|$ and this implies that

$$
\phi(x, \lambda)=O\left(e^{|t| x}\right)
$$

By the aid of (21) we find that

$$
\int_{0}^{x} \frac{\sin \lambda(x-\tau)}{\lambda} q(\tau) \phi(\tau, \lambda) d \tau=O\left(\frac{e^{|t| x}}{|\lambda|}\right) .
$$

From (14) and (21) it follows that, $\varphi(x, \lambda)$ has the asymptotic formula (19).

Theorem 5. Let $\lambda=\sigma+i t$ and suppose that $q(x)$ has a second order piecewise differentiable derivatives on $[0, \pi]$. Then the solution $\phi(x, \lambda)$ of nonlocal boundary value (1) and (2) have the following asymptotic formula

$$
\begin{aligned}
\phi(x, \lambda) & =\cos \lambda x+\frac{\alpha_{1}(x)}{\lambda} \sin \lambda x+\frac{\alpha_{2}(x)}{\lambda^{2}} \cos \lambda x+\frac{\alpha_{3}(x)}{\lambda^{3}} \sin \lambda x \\
& +O\left(\frac{e^{|t| x}}{\left|\lambda^{4}\right|}\right)
\end{aligned}
$$


where

$$
\begin{aligned}
\alpha_{1}(x) & =\frac{1}{2} \int_{0}^{x} q(t) d t+H \\
\alpha_{2}(x) & =-\frac{1}{4}\left(\int_{0}^{x} q(t) d t\right)^{2}+\frac{H}{2} \int_{0}^{x} q(t) d t+\frac{1}{4}[q(x)-q(0)], \\
\alpha_{3}(x) & =-\frac{1}{8}\left(\int_{0}^{x} q(t) d t\right)^{3}+\frac{H}{8}\left(\int_{0}^{x} q(t) d t\right)^{2}+\frac{H^{2}}{4} \int_{0}^{x} q(t) d t \\
& +\frac{H}{4}[q(x)-q(0)]+\frac{1}{8}\left[q^{\prime}(x)-q^{\prime}(0)\right] .
\end{aligned}
$$

Proof. By substituting from (19) into the integral equation (14), we have

$$
\begin{aligned}
\phi(x, \lambda) & =\cos \lambda x+\frac{H}{\lambda} \sin \lambda x+\frac{\sin \lambda x}{2 \lambda} \int_{0}^{x} q(t) d t \\
& +\frac{1}{2 \lambda} \int_{0}^{x} \sin \lambda(x-2 t) q(t) d t+O\left(\frac{e^{|\operatorname{Im} \lambda| x}}{|\lambda|^{2}}\right) .
\end{aligned}
$$

Integrating the last integration of (26) by parts and noticing that there exists $q^{\prime}(x)$ such that $q^{\prime} \in L_{1}[0, \pi]$

$$
\begin{gathered}
\frac{1}{2 \lambda} \int_{0}^{x} \sin \lambda(x-2 t) q(t) d t \\
=\frac{1}{4}[q(x)-q(0)] \frac{\cos \lambda x}{\lambda^{2}}-\frac{1}{4 \lambda^{2}} \int_{0}^{x} \cos \lambda(x-2 t) q^{\prime}(t) d t \\
=O\left(\frac{e^{|\operatorname{Im} \lambda| x}}{|\lambda|^{2}}\right),
\end{gathered}
$$

Substituting (27) in (26), we get

$$
\phi(x, \lambda)=\cos \lambda x+\frac{\alpha_{1}(x)}{\lambda} \sin \lambda x+O\left(\frac{e^{|\operatorname{Im} \lambda| x}}{|\lambda|^{2}}\right),
$$

where $\alpha_{1}(x)$ is defined by (25). In order to make $\phi(x, \lambda)$ more precise we repeat this procedure again by substituting from the last result (28) into the same integral equation (14), we have

$$
\phi(x, \lambda)=\cos \lambda x+\frac{H}{\lambda} \sin \lambda x+\int_{0}^{x} \frac{\sin \lambda(x-t) \cos \lambda t}{\lambda} q(t) d t
$$




$$
\begin{aligned}
& +\int_{0}^{x} \frac{\sin \lambda(x-t) \sin \lambda x}{\lambda^{2}} q(t) \alpha_{1}(t) d t \\
& +\int_{0}^{x} \frac{\sin \lambda(x-t)}{\lambda} q(t) O\left(\frac{e^{|\operatorname{Im} \lambda| x}}{|\lambda|^{2}}\right) d t .
\end{aligned}
$$

Now we estimate each term in (29). Integrating by parts twice the first term of (29), and noticing that $q^{\prime \prime} \in L_{1}[0, \pi]$, we have

$$
\begin{gathered}
\int_{0}^{x} \frac{\sin \lambda(x-t) \cos \lambda t}{\lambda} q(t) d t \\
=\frac{\sin \lambda x}{2 \lambda} \int_{0}^{x} q(t) d t+\frac{[q(x)-q(0)]}{4 \lambda^{2}} \cos \lambda x+O\left(\frac{e^{|\operatorname{Im} \lambda| x}}{|\lambda|^{3}}\right) .
\end{gathered}
$$

Similary, we have

$$
\begin{gathered}
\int_{0}^{x} \frac{\sin \lambda(x-t) \sin \lambda t}{\lambda^{2}} q(t) \alpha_{1}(t) d t \\
=-\frac{1}{2} \int_{0}^{x} \alpha_{1}(t) q(t) d t \frac{\cos \lambda x}{\lambda^{2}}+O\left(\frac{e^{|\operatorname{Im} \lambda| x}}{|\lambda|^{3}}\right) .
\end{gathered}
$$

Substituting (30) and (31) in (29), we get

$$
\phi(x, \lambda)=\cos \lambda x+\frac{\alpha_{1}(x)}{\lambda} \sin \lambda x+\frac{\alpha_{2}}{\lambda^{2}} \cos \lambda x+O\left(\frac{e^{|\operatorname{Im} \lambda| x}}{|\lambda|^{3}}\right)
$$

where $\alpha_{1}(x)$ and $\alpha_{2}(x)$ is defined by (25). In order to make $\phi(x, \lambda)$ more precise we repeat this procedure again by substituting from the last result (32) into the same integral equation (14), we have

$$
\begin{aligned}
\phi(x, \lambda)= & \cos \lambda x+\frac{H}{\lambda} \sin \lambda x+\int_{0}^{x} \frac{\sin \lambda(x-t) \cos \lambda t}{\lambda} q(t) d t \\
& +\int_{0}^{x} \frac{\sin \lambda(x-t) \sin \lambda t}{\lambda^{2}} q(t) \alpha_{1}(t) d t \\
& +\int_{0}^{x} \frac{\sin \lambda(x-t) \cos \lambda t}{\lambda^{3}} q(t) \alpha_{2}(t) d t \\
& +\int_{0}^{x} \frac{\sin \lambda(x-t)}{\lambda} q(t) O\left(\frac{e^{|\operatorname{Im} \lambda| x}}{|\lambda|^{4}}\right) d t .
\end{aligned}
$$


Now we estimate each term in (33). Integrating by parts twice the first term of (33), and noticing that $q^{\prime \prime} \in L_{1}[0, \pi]$, we have

$$
\begin{gathered}
\int_{0}^{x} \frac{\sin \lambda(x-t) \cos \lambda t}{\lambda} q(t) d t=\frac{\sin \lambda x}{2 \lambda} \int_{0}^{x} q(t) d t \\
+\frac{[q(x)-q(0)]}{4 \lambda^{2}} \cos \lambda x+\frac{\sin \lambda x}{8 \lambda^{3}}\left[q^{\prime}(x)-q^{\prime}(0)\right]+O\left(\frac{e^{|I m \lambda| x}}{|\lambda|^{4}}\right) .
\end{gathered}
$$

Further,

$$
\begin{gathered}
\int_{0}^{x} \frac{\sin \lambda(x-t) \sin \lambda t}{\lambda^{2}} q(t) \alpha_{1}(t) d t=-\frac{\cos \lambda x}{2 \lambda^{2}} \int_{0}^{x} \alpha_{1}(t) q(t) d t \\
+\frac{\sin \lambda x}{4 \lambda^{3}}\left[q(0) \alpha_{1}(0)-q(x) \alpha_{1}(x)\right]+O\left(\frac{e^{|\operatorname{Im} \lambda| x}}{|\lambda|^{3}}\right)
\end{gathered}
$$

and

$$
\begin{gathered}
\int_{0}^{x} \frac{\sin \lambda(x-t) \cos \lambda t}{\lambda^{3}} q(t) \alpha_{2}(t) d t \\
=\frac{\sin \lambda x}{2 \lambda^{3}} \int_{0}^{x} \alpha_{2}(t) q(t) d t+O\left(\frac{e^{|\operatorname{Im} \lambda| x}}{|\lambda|^{3}}\right) .
\end{gathered}
$$

Substituting from (34)-(36) into (33) we get the required formula (24).

Now inserting the values of the functions $\varphi(x, \lambda)$ from the estimate $(24)$ into the second of the boundary conditions in (2), we obtain the following equation for the determination of the eigenvalues equation (19) is the characteristic equation which gives roots of $\lambda$

$$
\lambda_{n}^{0}=\left(n+\frac{1}{2}\right) \frac{\pi}{\xi}, \quad n=0, \pm 1, \pm 2, \ldots .
$$

Then the $\omega(\lambda)$ has the same root of the function $\sin \lambda \xi$ (By Rouche's theorem)

$$
\lambda_{n}=\lambda_{n}^{0}+\varepsilon_{n}, \quad n=0,1,2, \ldots .
$$

Theorem 6. Let $q \in L_{1}(0, \pi)$, then we have the following asymptotic formulas for $\lambda_{n}$ of non-local boundary value (1) and (2)

$$
\lambda_{n}=\left(n+\frac{1}{2}\right) \frac{\pi}{\xi}+\frac{\alpha_{1}}{n \pi}+O\left(\frac{1}{n^{2}}\right),
$$

where $\alpha_{1}(x)$ defined in (25). 
Proof.

$$
\omega(x, \lambda)=\cos \lambda \xi+\frac{\alpha_{1}}{\lambda} \sin \lambda \xi+\frac{\alpha_{2}}{\lambda^{2}} \cos \lambda \xi+\frac{\alpha_{3}}{\lambda^{3}} \sin \lambda \xi+O\left(\frac{e^{|\operatorname{Im} \lambda| \xi}}{\left|\lambda^{4}\right|}\right)
$$

It follows from (39) that

$$
\cos \lambda \xi+\frac{\alpha_{1}}{\lambda} \sin \lambda \xi+\frac{\alpha_{2}}{\lambda^{2}} \cos \lambda \xi+\frac{\alpha_{3}}{\lambda^{3}} \sin \lambda \xi+O\left(\frac{e^{|\operatorname{Im} \lambda| \xi}}{\left|\lambda^{4}\right|}\right)=0
$$

From equation (40), we have

$$
\left[1+\frac{\alpha_{2}}{\lambda^{2}}\right] \cos \lambda \xi+\left[\frac{\alpha_{1}}{\lambda}+\frac{\alpha_{3}}{\lambda^{3}}\right] \sin \lambda \xi=0 .
$$

Dividing (41) by $\sin \lambda \xi$ we obtain

$$
\left[1+\frac{\alpha_{2}}{\lambda^{2}}\right] \cot \lambda \xi=-\left[\frac{\alpha_{1}}{\lambda}+\frac{\alpha_{3}}{\lambda^{3}}\right]
$$

since imaginary $\lambda=O\left(\frac{1}{n}\right)$, then

$$
\cot \lambda_{n} \xi=-\frac{\alpha_{1}}{\lambda_{n}}+\frac{\alpha_{1} \alpha_{2}}{\lambda_{n}^{3}}-\frac{\alpha_{3}}{\lambda_{n}^{3}}+O\left(\frac{1}{n^{4}}\right)
$$

from (37), (42) after elementary calculation, we obtain

$$
\varepsilon_{n}=\frac{\alpha_{1}}{n \pi}+O\left(\frac{1}{n^{2}}\right)
$$

From (37) and (43), we have

$$
\lambda_{n}=\left(n+\frac{1}{2}\right) \frac{\pi}{\xi}+\frac{\alpha_{1}}{n \pi}+O\left(\frac{1}{n^{2}}\right)
$$

Corollary 7. If $\xi=\pi$, then the eigenvalues of (38), we obtain

$$
\lambda_{n}=n+\frac{1}{2}+\frac{\alpha_{1}}{n \pi}+O\left(\frac{1}{n^{2}}\right) .
$$

Which meets with the result obtained in [9] 


\section{References}

[1] A.V. Bitsadze and A.A. Samarskii, Some elementary generalizations of linear elliptic boundary value problems, Dokl. Akad. Nauk SSSR, 185 (1969), 739-740.

[2] W.A. Day, Extensions of a property of the heat equation to linear thermoelasticity and order theories, Quart. Appl. Math., 40 (1982), 319-330.

[3] A.M.A. El-Sayed, E.M. Hamdallah, Kh.W. Elkadeky, Solution of a class of internal nonlocal Cauchy problems for the differential equation $x^{\prime}(t)=f\left(t, x(t), x^{\prime}(t)\right)$, Fixed Piont Theorem, 15, No. 2 (2014), 441-448.

[4] A.M.A. EL-Sayed, M.S. EL-Azab, A. Elsaid and S.M. Helal, Eigenvalue problem for Elliptic partial differential equations with nonlocal boudary conditions, Journal of Fractional Calculus and Applications, 5, No. 14 (2014), 1-11.

[5] A.M.A. EL-Sayed and F.M. Gaafar, Stability of a nonlinear non-autonomous fractional order systems with different delays and non-local conditions, Advances in Difference Equations (2011), doi: 10.1186/1687-1847-2011-47.

[6] A.M.A. EL-Sayed, Z.F.A. EL-Raheem and N.A.O. Buhalima, Eigenvalues and eigenfunctions of non-local boundary value problems of the Sturm-Liouville equation, Electronic Journal of Mathematical Analysis and Applications, 5, No. 1 (2017), 179-186.

[7] A.M.A. EL-Sayed, Z.F.A. EL-Raheem and N.A.O. Buhalima, Eigenvalues and eigenfunctions of a non-local boundary value problem of Sturm-Liouville diferential equation, Global Journal of Pure and Applied Mathematics, 12, No. 5 (2016), 3885-3893.

[8] A.M.A. EL-Sayed, Z.F.A. EL-Raheem and N.A. Buhalima, Eigenvalues and eigenfunctions for reqular Sturm-Liouville equation with non-local boundary conditions, International Journal of Pure and Applied Mathematics, 112, No. 1 (2017), 103-113.

[9] G. Freiling, V. Yurko, Inverse Sturm-Liouville Problems and their Applications, Nova Science, New York, 2001.

[10] N. Gordeziani, On some non-local problems of the theory of elasticity, Buletin of TICMI, 4 (2000), 43-46.

[11] N.I. Ionkin, The solution of a certain boundary value problem of the theory of heat conduction with a nonclassical boundary condition, Differ. Equ., 13, No. 2 (1977), 294304, In Russian.

[12] A. M. Nakhushev, Equations of Mathematical Biology, Vysshaya Shkola, Moscow, 1995, In Russian.

[13] K. Schuegerl, Bioreaction Engineering. Reactions Involving Microorganisms and Cells, Volume 1, John Wiley and Sons, 1987. 
